\title{
The jet of the BL Lacertae object PKS 0521-365 in the near-IR: MAD adaptive optics observations ${ }^{\star}$
}

\author{
R. Falomo ${ }^{1}$, E. Pian ${ }^{2}$, A. Treves ${ }^{3}$, G. Giovannini ${ }^{4,5}$, T. Venturi ${ }^{4}$, A. Moretti ${ }^{1}$, C. Arcidiacono ${ }^{1}$, \\ J. Farinato ${ }^{1}$, R. Ragazzoni ${ }^{1}$, E. Diolaiti ${ }^{6}$, M. Lombini ${ }^{6}$, F. Tavecchio ${ }^{7}$, R. Brast ${ }^{5}$, R. Donaldson ${ }^{8}$, \\ J. Kolb ${ }^{8}$, E. Marchetti ${ }^{8}$, and S. Tordo ${ }^{8}$ \\ 1 Osservatorio Astronomico di Padova, INAF, vicolo dell'Osservatorio 5, 35122 Padova, Italy \\ e-mail: renato.falomo@oapd.inaf.it \\ 2 Osservatorio Astronomico di Trieste, INAF, via Tielopo, Trieste, Italy \\ 3 Universita' dell'Insubria (Como), Italy \\ 4 Istituto di Radioastronomia, INAF c/o CNR, via Gobetti 10140129 Bologna, Italy \\ 5 Dipartimento di Astronomia - Bologna University - via Ranzani 140127 Bologna, Italy \\ ${ }^{6}$ Osservatorio Astronomico di Bologna, INAF, via Ranzani 1, 40127 Bologna, Italy \\ 7 Osservatorio Astronomico di Brera, INAF, via Bianchi 46, Merate, Italy \\ 8 European Southern Observatory, Karl-Schwarzschild-Str. 2, 85748 Garching bei München, Germany
}

Received 16 March 2009 / Accepted 17 April 2009

\section{ABSTRACT}

Context. BL Lac objects are low-power active nuclei exhibiting a variety of peculiar properties caused by the presence of a relativistic jet and orientation effects.

Aims. We present adaptive optics near-IR images at high spatial resolution of the nearby BL Lac object PKS 0521-365, which is known to display a prominent jet both at radio and optical frequencies.

Methods. The observations were obtained in Ks-band using the ESO multi-conjugated adaptive optics demonstrator at the Very Large Telescope. This allowed us to obtain images with 0.1 arcsec effective resolution. We performed a detailed analysis of the jet and its related features from the near-IR images, and combined them with images previously obtained with HST in the $R$ band and by a re-analysis of VLA radio maps.

Results. We find a remarkable similarity in the structure of the jet at radio, near-IR, and optical wavelengths. The broad-band emission of the jet knots is dominated by synchrotron radiation, while the nucleus also exhibits a significant inverse Compton component. We discovered the near-IR counterpart of the radio hotspot and found that the near-IR flux is consistent with being a synchrotron emission from radio to X-ray wavelengths. The bright red object (red-tip), detached but well aligned with the jet, is well resolved in the near-IR and has a linear light profile. Since it has no radio counterpart, we propose that it is a background galaxy not associated with the jet. Conclusions. The new adaptive optics near-IR images and previous observations at other frequencies allow us to study the complex environment around the remarkable BL Lac object PKS 0521-365. These data exemplify the capabilities of multi conjugate adaptive optics observations of extragalactic extended sources.

Key words. galaxies: BL Lacertae objects: individual: PKS 0521-365 - instrumentation: adaptive optics

\section{Introduction}

BL Lac objects are a class of low-power active galactic nuclei (AGN) characterized by a lack or extreme weakness of emission lines observed in all other types of AGN. They also exhibit strong and rapid flux variability and significant polarization. The emission is dominated by a non-thermal component extending from radio to very high energies. These active nuclei are hosted by massive elliptical galaxies that appear in most cases unperturbed and located in moderately rich environments. A widely accepted model that explains these properties is based on the idea that these nuclei emit a relativistic jet oriented close to the line of sight of the observer. A direct consequence of this interpretation is that there are several other objects intrinsically identical to BL Lacs but with a misaligned jet that thus exhibits different nuclear properties. This parent population has been

\footnotetext{
^ Based on observations collected at ESO, Paranal, Chile, as part of MAD Guaranteed Time Observations.
}

identified with low-power radio galaxies (e.g., Urry \& Padovani 1995).

Since the jet is closely aligned with the line of sight, it is very difficult to observe unless the angular resolution is high. In the radio band, jet detection is indeed possible and a large fraction of objects classified as BL Lacs show a signature of a jet, often with evidence of superluminal motion e.g. Homan et al. 2001; Giroletti et al. 2004). On the other hand, the jet is rarely detected in the optical and X-ray bands. This depends both on the more limited angular resolution and on the short lifetime of the high energy electrons producing the non-thermal emission of the jet.

The first systematic searches for angularly resolved multiwavelength counterparts of radio jets in AGNs were carried out following the advent of high resolution imaging facilities such as HST and Chandra. The individual knots within the jets of M 87 and 3C273 have been studied in detail at optical and X-ray frequencies (e.g., Sparks et al. 1994; Marshall et al. 2001, 2005; Jester et al. 2007), and in M 87, evidence of knot variability has been found in both optical and X-ray bands (Perlman 2003; 
Harris et al. 2003, 2006). Jets of higher redshift radio-loud objects have been detected and resolved with HST and Chandra. About 20 blazars have been found to have optical and X-ray resolved jets to date (e.g., Scarpa et al. 1999; Schwartz et al. 2000; Sambruna et al. 2002, 2004, 2007, 2008; Marshall et al. 2005; Tavecchio et al. 2007). A detailed study of the optical jet based on HST images was completed for: PKS 0521-365, PKS 2201+04 and 3C 371 (Scarpa et al. 1999).

Stronger evidence of a prominent optical jet in a BL Lac object has been found for PKS 0521-365, and in this paper we present high resolution near-IR images of the source obtained at ESO-VLT. The new images were acquired using an adaptive optics (AO) camera, built to probe the new capabilities of the technique, and a re-analysis of the VLA radio data allow us to investigate accurately the properties of the jet and the surrounding structures.

We adopt the concordance cosmology with $H_{0}=$ $70 \mathrm{~km} \mathrm{~s}^{-1} \mathrm{Mpc}^{-1}, \Omega_{m}=0.3$, and $\Omega_{\Lambda}=0.7$.

\section{PKS 0521-365}

The radio source PKS 0521-365 is a well-studied nearby object $(z=0.0554$; Danziger 1979). It is one of the most remarkable extragalactic objects of the southern sky, because it exhibits a variety of nuclear and extra-nuclear phenomena. First classified as an $N$ galaxy, and then as a BL Lac object, it shows the strong narrow and broad emission lines typical of type 1 active nuclei both in optical and UV (Ulrich 1981; Danziger 1983; Scarpa 1995). Interestingly, the source has a prominent radio, optical, and X-ray jet (Danziger 1979; Keel 1986; Falomo 1994; Macchetto et al. 1991; Scarpa et al. 1999; Birkinshaw et al. 2002), which resembles that of the nearby radio galaxy M 87 (Sparks et al. 1994). Tingay et al. (1996, 1998), Tingay \& Edwards (2002) and Giroletti et al. (2004) studied the structure of this source on sub-pc scales in the radio.

The optical jet was observed with HST by Macchetto et al. (1991) and by Scarpa et al. (1999), who found a structure consisting of a bright knot close to the nucleus and a diffuse and knotty structure that extends out to $\sim 6$ arcsec from the nucleus. Farther out along the jet (but well separated), at $\sim 9$ arcsec, there is resolved compact emission (also called the "red tip") (Falomo 1994; Scarpa et al. 1999).

The overall energy distribution was discussed by Pian et al. (1996), who proposed that the jet is oriented at about 30 degrees with respect to the line of sight.

\section{Observations and data reduction}

\subsection{MAD near-IR AO images}

Near-infrared images of the target were obtained using the ESO Multi conjugate Adaptive optics Demonstrator (MAD). MAD is an experiment devoted to demonstrating the feasibility of the Multi Conjugate Adaptive Optics (MCAO) technique as a testbench for the European Extremely Large Telescope. MAD was mounted onboard the UT-3 Very Large Telescopes (VLT) and accomplished the first MCAO on-sky observations. MAD successfully implemented two MCAO techniques: the star oriented technique based on three Shack-Hartmann wavefront sensors and the layer oriented technique (LO) (Ragazzoni 2000; Ragazzoni et al. 2000) based on a multi pyramid sensor (Ragazzoni 1996). Both devices can use reference stars on a 2 arcmin technical Field of View; in particular, in the LO mode up to 8 pyramids can be positioned where the focal plane images of the reference stars

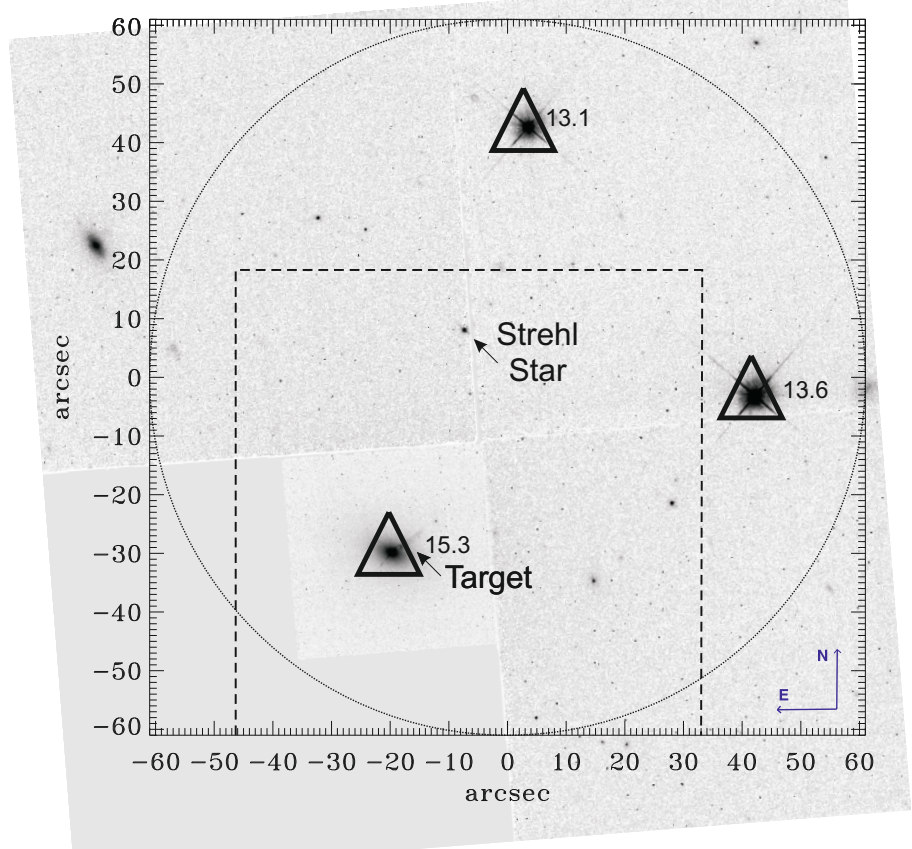

Fig. 1. The 2 arcmin field of view (circle) corrected by the MAD MCAO system. The area inside the dashed box was imaged by the NIR camera. The greyscale image is a mosaic of HST + WFPC 2 images ( $F 702 w$ filter). The triangles correspond to the LO AO reference stars. $R$-magnitude is reported for each star. At the centre of the field the arrow identifies the star used to compute the SR (see Table 1).

form, splitting the light into four beams. A beam splitter illuminates two identical objectives conjugated at different atmospheric altitudes. These optics coadd the pupil images of all reference stars on two fast readout CCDs simultaneously. These measurements are the input for computing the corrections to be applied to the two deformable mirrors, which are conjugated to zero and $8.5 \mathrm{~km}$ altitude. The observations presented here were obtained using the LO wavefront sensor only.

The images were obtained in a MCAO configuration with both deformable mirrors, and used two reference stars ( $R$-band magnitudes: 13.6, 13.0) and the core of the target $(R=15.3)$ to perform wavefront sensing (see Fig. 1). These correspond to an overall integrated magnitude of $R \sim 12.5$. Given that MAD wavefront sensing CCD sensitivity peaks in the $V$ band, we estimate an overall $V \sim 12$ integrated magnitude using colours of stars available from USNO.

In contrast to MCAO observations carried out in crowded regions, such as a Globular Cluster (Moretti et al. 2009; Bono et al. 2009), this observation demonstrates that the case where MCAO allows not only for a uniform and yet higher Strehl Ratio, but makes observations possible at about diffraction limit resolution, because of the increasing of the AO sky coverage. In fact, given the distance of the neighbourhoods stars from the target any Single Conjugated AO observations could use the object itself as a reference source. In this MCAO case the gain in terms of useful flux to achieve the AO compensation is more than an order of magnitude.

The observations were carried out with the telescope pointing close to the zenith, with airmass 1.05, and the ESO DIMM seeing monitor measured an average seeing in $V$ band of $1.1 \pm$ 0.1 arcsec. We computed the $F W H M$ and the strehl ratio (SR) 
Table 1. Journal of MAD observations.

\begin{tabular}{llcccc}
\hline \hline Dataset & Date & Seeing & $T_{\exp }$ & $F W H M$ & SR \\
\hline a) & $27 / 9 / 2007$ & 0.99 & 900 & 0.11 & 14.4 \\
b) & $27 / 9 / 2007$ & 1.02 & 900 & 0.12 & 26.3 \\
c) & $27 / 9 / 2007$ & 1.11 & 900 & 0.09 & 22.0 \\
d) & $27 / 9 / 2007$ & 1.05 & 900 & 0.10 & 15.9 \\
All & $27 / 9 / 2007$ & 1.04 & 3600 & 0.12 & 17.6 \\
\hline
\end{tabular}

(see Table 1), using a relatively bright star located near the centre of the field of view (see Fig. 1).

Images of the target were obtained following a 5-position jitter pattern, with offsets of 5 arcsec, to ensure an adequate subtraction of the sky background. The entire data set consists of 20 exposures with slightly different positions with the pattern, for a total exposure time of $3600 \mathrm{~s}$.

Each individual image was trimmed and flat fielded with the appropriate flat-field frame derived from several images obtained on the sky at the beginning of the night in the $K_{s}$ filter. A bad-pixel mask was created by analysing the ratio of two flatfield images with significant different exposure levels to identify the pixels that not responded adequately to the signal intensity.

For each observation, we constructed a reference sky image, which was subtracted from the science frame to obtain the net intensity frame. This is particularly important for NIR observations since they are dominated by the sky emission. The sky variation was $\sim 2 \%$. This allowed us to construct a sky-background frame from the median of all science frames gathered for each target. The final background frame was normalized to the median counts of each scientific frame before being subtracted.

Finally, we combined all sky-subtracted and registered frames into a final image. In Table 1 , we list the total exposure time of the images that have been effectively used to construct the final image. As a first measure of the quality of the photometry, we also list the average $F W H M$ measured in each combined frame.

\subsection{HST optical images}

PKS 0521-365 was observed with HST using WFPC2 in the $R(F 702 W)$ filter as part of the HST snapshot survey of BL Lac objects (Scarpa et al. 2000; Urry et al. 2000). The target was imaged with the PC device for a total exposure time of $305 \mathrm{~s}$. These images were discussed in detail in Scarpa et al. (1999) and are used here to compare with NIR data obtained with MAD at VLT.

\section{3. $V L A$ radio data and morphology}

VLA radio observations at 1.4 and $15 \mathrm{GHz}$ were retrieved from archive data. Both observations were taken with the array in the BnA configuration. The $1.4 \mathrm{GHz}$ observations were gathered on July 1987 (Project ID AV151) and those at 15 GHz on March 1985 (Project ID AL508). PKS 0521-365 was observed for only $2 \mathrm{mn}$ at $1.4 \mathrm{GHz}$, while the $15 \mathrm{GHz}$ observations had a total time on source of $2.5 \mathrm{~h}$.

Published images are available at both frequencies $(1.4 \mathrm{GHz}$ Keel 1984; 15 GHz, Keel et al. 1986), although to take advantage of today's more powerful computer and data reduction facilities, we re-analysed both data sets. To obtain our final images, we calibrated the data with the present standard procedures in AIPS, accurately edited the visibilities, and carried out a few self- calibration cycles: i.e., 4 in phase only for $1.4 \mathrm{GHz}$ data and 2 in phase and one more in phase and gain for $15 \mathrm{GHz}$ data. The final
Table 2. Parameters of the radio images.

\begin{tabular}{lcc}
\hline \hline $\begin{array}{l}\text { Frequency } \\
\text { GHz }\end{array}$ & $\begin{array}{c}H P B W \\
\text { (degree) }\end{array}$ & $\begin{array}{c}\text { rms } \\
\text { mJy/beam }\end{array}$ \\
\hline 1.47 & $4.11 \times 1.21(-7)$ & 0.6 \\
15.11 & $0.6 \times 0.6$ & 0.25 \\
15.11 & $0.3 \times 0.3$ & 0.28 \\
\hline
\end{tabular}

Column 1: Observing frequency, Col. 2: HPBW and Position Angle of the major axis in degree; Col. 3: $1 \mathrm{rms}$ level.

images were produced with the IMAGR task. The parameters of the images discussed here are given in Table 2.

In Fig. 2, we show a full resolution $(H P B W=0.3 \operatorname{arcsec})$ $15 \mathrm{GHz}$ image with radio contours overlaid on the near-IR image. The radio source is dominated by a bright core with a peak flux density of 2.6 Jy. The well known one-sided jet (Keel 1986) is clearly visible on the N-W side, while on the opposite side a bright hot spot is present. No indication of a hot spot is visible on the side of the main radio jet. At lower resolution (Fig. 2), the $\mathrm{W}$ lobe is marginally visible with faint substructures. The eastern lobe has a higher brightness and exhibits a morphology typical of FR II sources, where the lobe fills the region between the core and the hot spot. The lobe structure is in agreement with a backflow of the radio plasma after a strong interaction between the hot spot at the end of the relativistic jet and the surrounding medium.

\section{Results}

In Fig. 2, we show the near-IR image of PKS 0521-365 obtained by MAD compared with the radio map at $15 \mathrm{GHz}$. At both nearIR and radio wavelengths, the jet is clearly well detected together with the nucleus and the bright knots. On the opposite side of the jet, there is fainter emission within the MAD image which is coincident with the radio hot spot.

\subsection{Analysis and measurements of the jet}

To enhance the structure of the jet in the near-IR image, we have subtracted the emission of both the nucleus and the host galaxy from the original image. To subtract the host galaxy, we performed a detailed two-dimensional surface photometry analysis of the source and derive a clean model of the object. The adopted procedure is very similar to that described in Falomo et al. (2000). The emission from the jet after the subtraction of the nucleus and the host galaxy is shown in Fig. 4. The bright knot $\mathrm{A}$ at 1.9 arcsec from the nucleus and the diffuse emission from the jet are clearly detected in near-IR as well as the resolved feature in the red-tip. In Fig. 5, we show the brightness profile along the jet.

To compare the features in NIR, optical, and radio bands, we applied the same procedure used for the MAD image to subtract the host galaxy and the nucleus from the HST $R$ band image. In Fig. 3 we compare images of the jet in the three different bands. The overall morphology of the jet in the near-IR is similar to that observed in the optical (Scarpa et al. 2000) and at $2 \mathrm{~cm}$ (Keel 1986). The general shape and total length of the radio jet are almost exactly reproduced in the near-IR, in the optical, and at radio frequencies.

Following the previous analysis of the jet, 4 knots can be easily recognized. These have been labelled in Fig. 3, according the definitions introduced in Falomo (1994). To construct the spectral emission of these components of the jet, we measured 


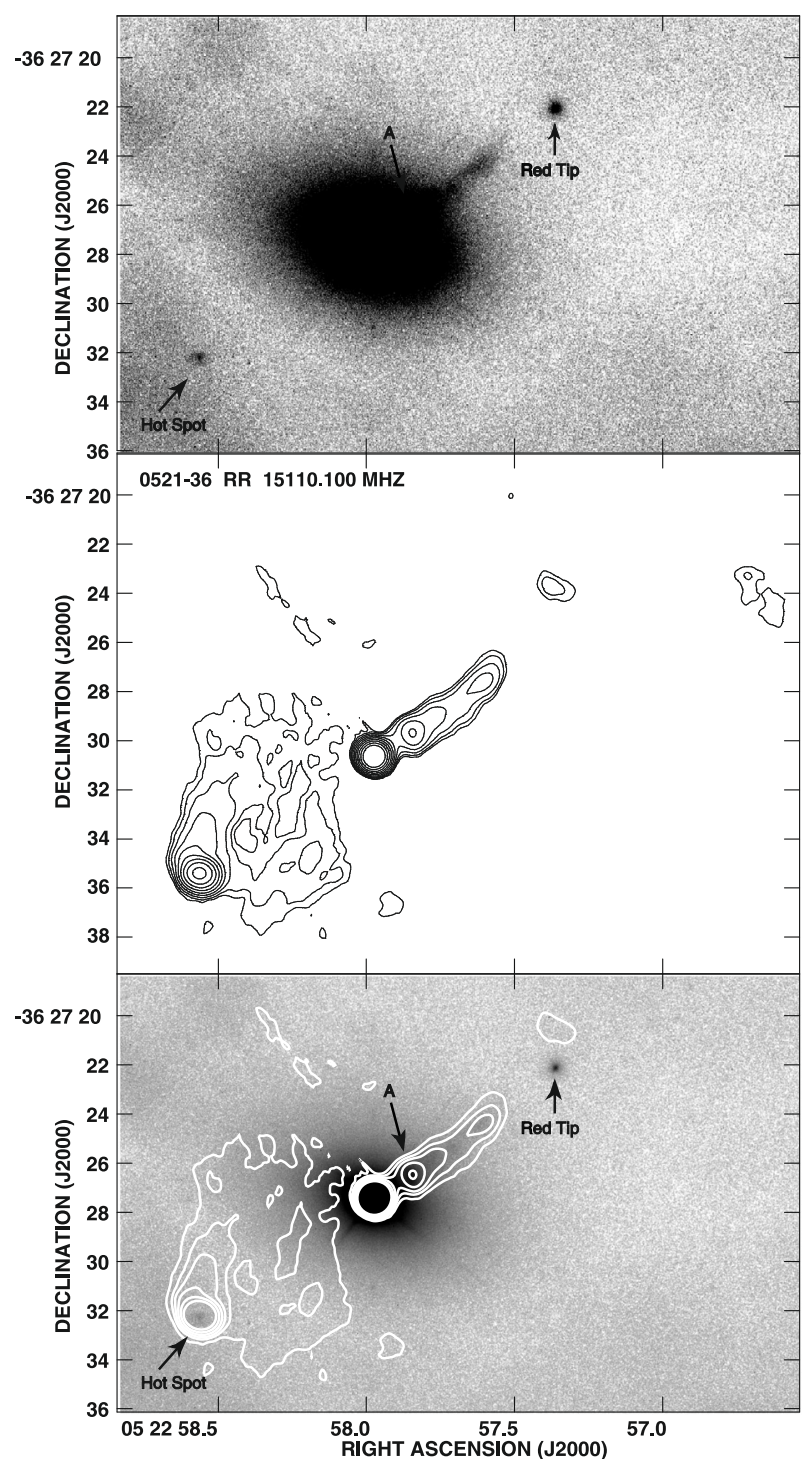

Fig. 2. Upper panel: the image of PKS 0521-365 observed by MAD in the $K \mathrm{~s}$ band. Middle panel: the contours represent the VLA radio map at $15 \mathrm{GHz}$. Contour levels in the middle panel are: 1,4,8,16,32,64 mJy/beam. Bottom panel: contour levels in the bottom panel are: $-1,1,2,4,8,16,32,64,128,256,512 \mathrm{mJy} /$ beam $(0.6 \times 0.6 \operatorname{arcsec})$.

the flux of the four knots by adopting a fixed square aperture of 1 arcsec. In Table 3, we report the positions and the measurements of the knots in all bands. In the radio band, the knots are well resolved with sizes given in Table 3.

\subsection{The nature of the red tip}

The near-IR image obtained by MAD also clearly detects and resolves the diffuse emission at about 9 arcsec NW from the nucleus (see Table 3 for details). The observed apparent mag in $K=17.9$. The object is very red: $R-K=3.4$ (using $R_{\text {mag }}=21.3$ derived from HST image). This feature is well resolved in the near-IR and has an average surface brightness that is well described by a linear (disk like) profile.

We performed a detailed analysis of the radio emission in the region around the red tip. This revealed extended radio emission at about $(\mathrm{RA}=0522$ 57.38; Dec $=-3627$ 23.8) that is not exactly coincident with the optical position (RA = 0522 57.3; Dec $=-362725.5$ ) of the red tip but sufficiently close to be
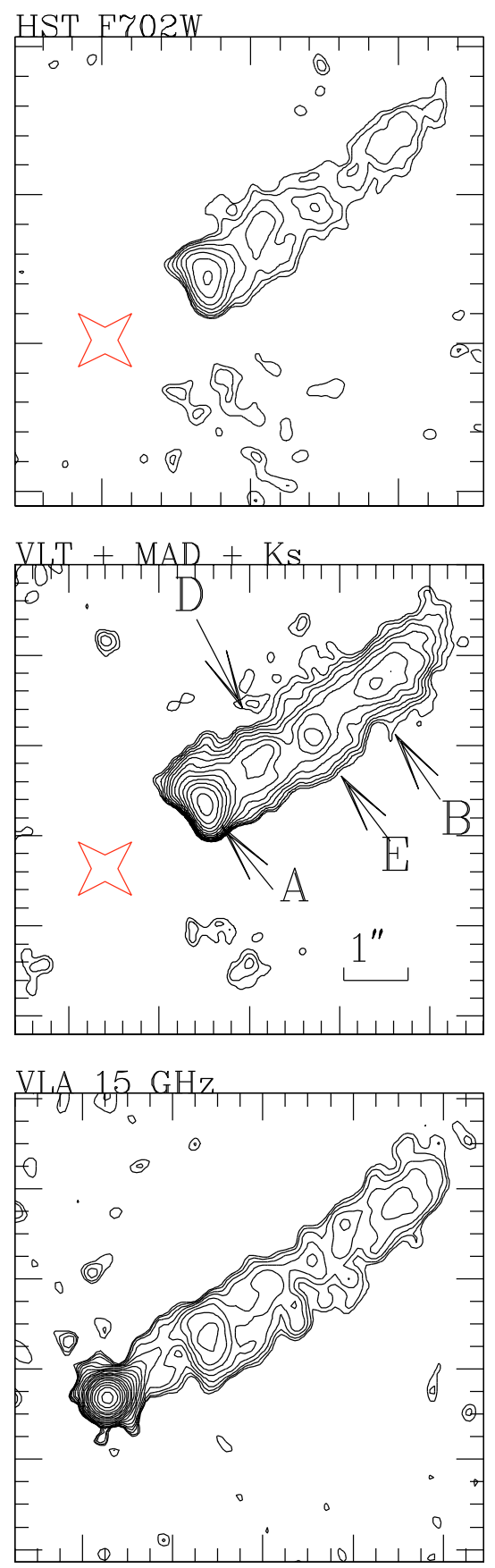

Fig. 3. Upper panel: The contour plot of the jet of PKS 0521-365 observed by MAD in the $K$ s band (middle panel) compared with the image of the jet observed in $R$ band by HST+ WFPC2 (top panel) and the radio map at $15 \mathrm{GHz}$ obtained by VLA (bottom panel). The large star represents the position of the (subtracted) nucleus in both optical and near-IR bands. In the bottom panel, the radio jet is shown at the same angular resolution as the near-IR data. The nuclear source has not been subtracted. A collimated jet with the same position angle as the VLBI pc-scale jet is present near the core. The jet becomes resolved transversally at less than $1^{\prime \prime}$ from the core.

considered physically associated. The shape of this radio structure suggests that it has the characteristic morphology of a headtail radio galaxy. Based on these coincidences we argue that the red tip could be a background, faint radio-galaxy. 
Table 3. Jet measurements of PKS 0521-365.

\begin{tabular}{cccccc}
\hline \hline Feature & $\begin{array}{c}K_{\mathrm{s}}^{a} \\
(\mathrm{mag})\end{array}$ & $\begin{array}{c}R^{b} \\
(\mathrm{mag})\end{array}$ & $\begin{array}{c}F_{\text {Radio }}^{c} \\
(\mathrm{mJy})\end{array}$ & $\begin{array}{c}\text { Size }^{d} \\
(\operatorname{arcsec})\end{array}$ & $\begin{array}{c}F_{\mathrm{X}-\mathrm{ray}}^{e} \\
(\mathrm{mJy})\end{array}$ \\
\hline Nucleus & $11.9 \pm 0.05$ & $15.30 \pm 0.05$ & $2600 \pm 100$ & $\ldots$ & $(6.8 \pm 0.1) \times 10^{-4}$ \\
A & $17.6 \pm 0.1$ & $20.3 \pm 0.1$ & $58 \pm 5$ & $0.7 \times 0.5$ & $(14 \pm 3) \times 10^{-6}$ \\
D & $18.4 \pm 0.2$ & $21.6 \pm 0.2$ & $20 \pm 3$ & $\ldots$ & $\ldots$ \\
E & $18.5 \pm 0.2$ & $22.1 \pm 0.2$ & $14 \pm 2$ & $\ldots$ & $\ldots$ \\
B & $18.5 \pm 0.2$ & $22.0 \pm 0.2$ & $19 \pm 3$ & $1.3 \times 0.6$ & $\ldots$ \\
red-tip & $17.9 \pm 0.2$ & $21.3 \pm 0.2$ & $\ldots$ & $0.6 \times 0.4$ & $\ldots$ \\
hotspot & $19.9 \pm 0.2$ & $>22$ & $588 \pm 6$ & $\ldots$ & $\approx 4 \times 10^{-7}$ \\
\hline
\end{tabular}

${ }^{a} K_{s}$-band magnitude from VLT MAD measurements; ${ }^{b} R$-band magnitude from HST WFPC2 measurements in $F 702 W$ filter, not corrected for Galactic reddening; ${ }^{c}$ radio measurements at $15 \mathrm{GHz}$ from VLA high resolution map; ${ }^{d}$ measured on the radio maps; ${ }^{e} \mathrm{X}$-ray measurements at $1 \mathrm{keV}$ from Chandra images, corrected for Galactic absorption (Birkinshaw et al. 2002).

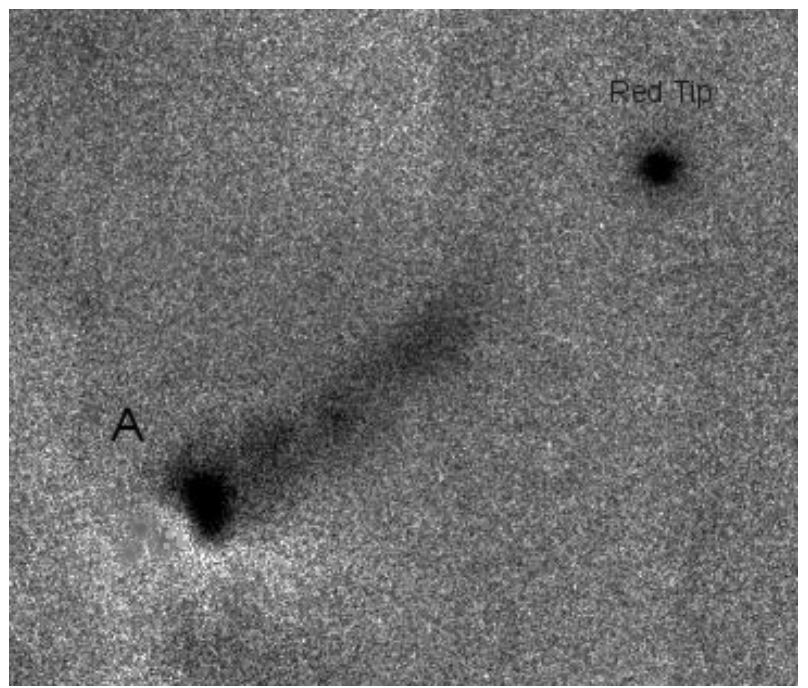

Fig. 4. The near-IR image of the jet of the BL Lac object PKS 0521365 , as observed by MAD at VLT in Ks filter. The host galaxy and the nucleus have been subtracted from the original image (see text). The field of view shown is 10 arcsec.

\subsection{The near-IR counterpart of the radio hot spot}

In the $1.4 \mathrm{GHz}$ image (see Fig. $6 ; H P B W=4.11^{\prime \prime} \times 1.21^{\prime \prime}$ in PA 7 ), the hot spot is the dominant feature (peak flux $=2.8 \mathrm{Jy} /$ beam) because of its steep spectrum, while the core is fainter (1.8 Jy) with respect to the $15 \mathrm{GHz}$ flux density, which is self-absorbed. This image confirms the lack of a hot spot like structure on the jet side. At $1.4 \mathrm{GHz}$, the radio emission is extended westwards, with a low brightness morphology typical of FR I sources, while the East lobe shows a large extension in N-S but always in the backflow region in-between the core and the hot spot. No indication of a counter-jet is visible.

In our near-IR image we searched for emission that is coincident with the radio hot spot and found a faint $\left(K_{S} \sim 20\right)$ compact feature at the position RA $=05: 22: 58.54 \mathrm{Dec}=-36: 27: 35.67$ (Equinox: 2000.0). This is coincident with the peak emission of the hot spot to within 0.2 arcsec. At this position, there is no optical counterpart in the $R$-band HST image. We estimate a magnitude limit of $R>22$ for this feature. In a few other cases, the optical-NIR counterpart of radio hot-spots was detected (e.g. Dreher et al. 1986; Hartman et al. 1999; Meisenheimer 1997; Hardcastle 2001; Prieto et al. 2003).

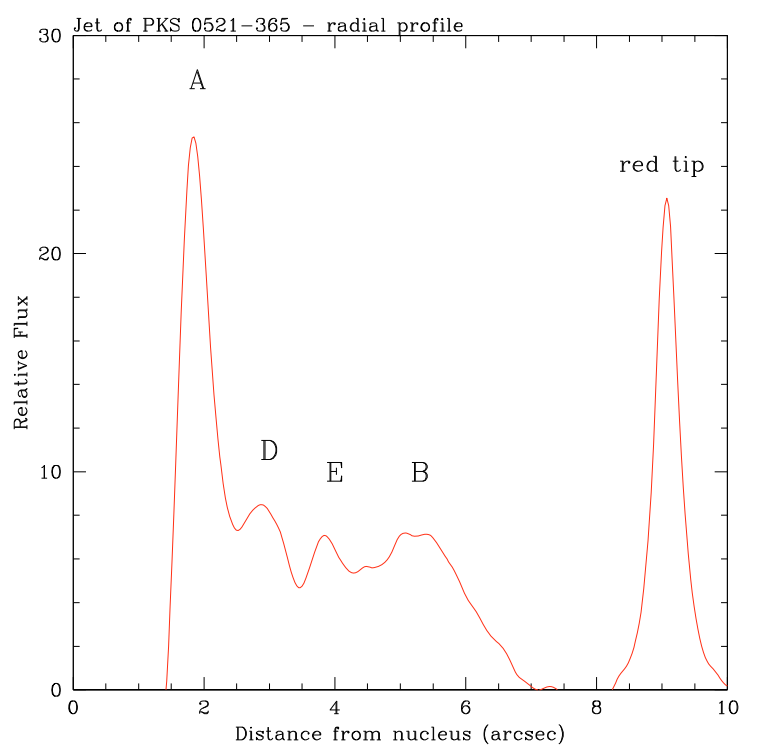

Fig. 5. The brightness profile along the jet of the BL Lac object PKS 0521-365 and of the red-tip as observed by MAD at VLT in Ks filter. The profile was obtained by integrating the signal in a strip of fixed size of 0.56 arcsec width between the nucleus and the red-tip. The main peaks correspond to the knots labelled in Fig. 3. The flux from both the nucleus and the host galaxy has been removed.

\section{Discussion}

\subsection{Spectral energy distribution of jet components}

We have combined our near-IR measurements of the nucleus, jet knots, and hotspot with the available optical, radio and X-ray fluxes to construct overall spectral energy distributions (SED) of main components. The optical and near-IR fluxes were corrected for Galactic dust absorption using $E_{B-V}=0.039$ (Schlegel et al. 1998), and the (Cardelli et al. 1989) extinction curve. The X-ray fluxes (Birkinshaw et al. 2002) were corrected for Galactic absorption assuming $N_{\mathrm{H}}=3.37 \times 10^{20} \mathrm{~cm}^{-2}$. The resulting spectral energy distributions are reported in Fig. 7.

The SED of the core has been compared (assuming modest multiwavelength variability) with a classical synchrotron emission model in a single region of radius $R_{0}$ filled homogeneously with a magnetic field $\mathrm{B}$, in equipartition with the relativistic particles, following the formalism of Ghisellini et al. (1998) and Sari et al. (1998). In the emitting region, the electrons are accelerated by a relativistic shock to a power-law distribution with a minimum Lorentz factor $\gamma_{\mathrm{m}}: N\left(\gamma_{\mathrm{e}}\right) \propto \gamma_{\mathrm{e}}^{-p} \mathrm{~d} \gamma_{\mathrm{e}}$, with $\gamma_{\mathrm{e}} \geq \gamma_{m}$. The 


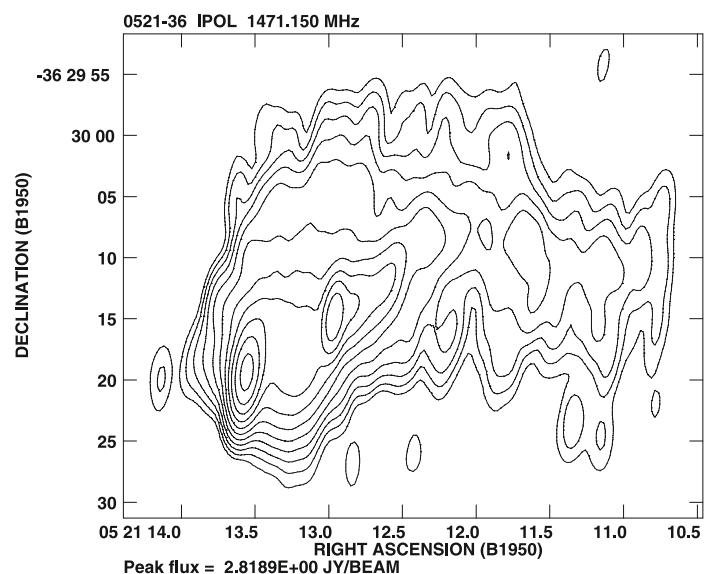

Fig. 6. The radio map at $1.4 \mathrm{GHz}$ of PKS 0521-365. Contour levels are: $-2,2,4,8,16,32,64, \ldots, 2048 \mathrm{mJy} / \mathrm{b}$. The beam size is $4.11 \times 1.21$ (arcsec); p.a. -7 .

spectral power peaks at the cooling frequency $v_{\mathrm{c}}$, corresponding to the electron energy $\gamma_{\mathrm{c}}$ above which the electron radiative losses become significant (note that in Ghisellini et al. 1998) this is named $\left.\gamma_{\min }\right)$. The spectral index $\alpha_{0}$ below $v_{\mathrm{c}}\left(F_{v} \propto v^{-\alpha}\right)$ is related to the electron distribution index by $p=2 \alpha_{0}+1$. The spectrum above $v_{\mathrm{c}}$ has an index $\alpha_{1}=\alpha_{0}+0.5$. In the case of the jet knots and hotspot, we found that this simple synchrotron model is not satisfactory for the description of the SED, therefore we adopted a curved electron distribution similar to that used in Tavecchio et al. (2002). In this formulation, the break random Lorentz factor $\gamma_{\mathrm{b}}$ corresponds to $\gamma_{\mathrm{c}}$ in the above simpler model.

In spite of the modest coverage of the points, most of the model parameters are well constrained by the data. First of all, the radio VLA and VLBI data suggest that the kinematic conditions in the jet of PKS 0521-365 are not extremely relativistic (Pian et al. 1996; Tingay \& Edwards 2002; Giroletti et al. 2004), which implies a moderate bulk Lorentz factor for the plasma motion in the jet (see also Birkinshaw et al. 2002). Second, the fact that an arcsec-scale jet is observed at all frequencies with a projected size of $\sim 7 \mathrm{kpc}$ indicates that our viewing angle is not small, so that the light aberration is not relevant. As proposed by Pian et al. (1996), we assume $\Gamma=1.2$ for all jet components (for the hotspot we assume $\Gamma=1.1$, to account for moderate plasma braking at that distance from the jet apex) and a jet viewing angle of 30 degrees. These parameters imply a relativistic Doppler factor $\delta=1.6$.

\section{The jet core}

For the nucleus, the size of the emitting region is constrained by the observed minimum variability timescale of about 1 day. The cooling frequency is probably below the infrared frequencies, which we set to be $v \sim 10^{14} \mathrm{~Hz}$. The IR/optical spectrum has a slope $\alpha_{1}=1.4$ (see also Pian et al. 1996), from which we derive $\alpha_{0}=0.9$. By assuming a magnetic field and electron density similar to those reported in Pian et al. (1996) - which appears reasonable considering the lack of substantial long-term multiwavelength variability - we obtain a good fit to the spectrum up to X-ray energies. However, the model does not reproduce satisfactorily the Chandra spectrum (see Fig. 7): this is clearly due to an extra component, which we identify with inverse Compton scattering of relativistic electrons off the synchrotron photons or off external radiation fields. A bright inverse

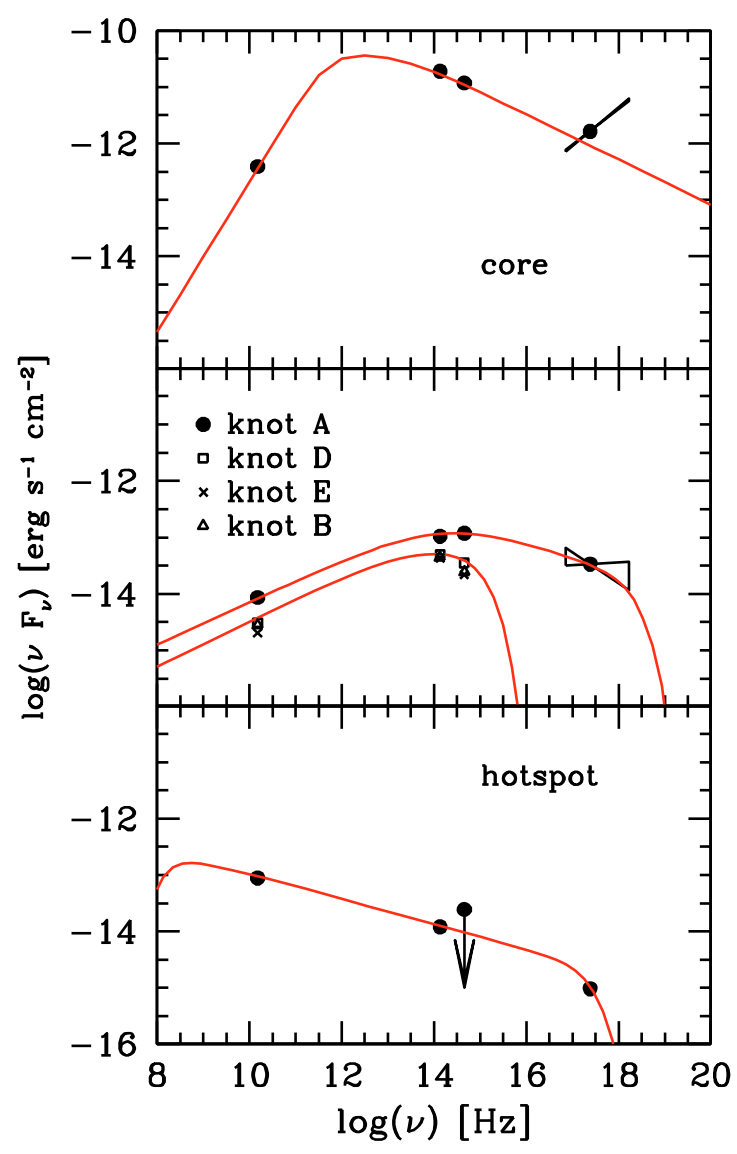

Fig. 7. The spectral energy distributions of the different components of the BL Lac object PKS 0521-365 ( $z=0.055)$ : nucleus (top), jet knots A,D,E,B (middle) and hot spot (bottom). All X-ray data are from Birkinshaw et al. (2002), and are corrected for the absorption of Galactic neutral hydrogen with a column density of $3.37 \times 10^{21} \mathrm{~cm}^{-2}$. The optical data of the nucleus and of the jet knot are measured from the HST image of Scarpa et al. (1999). The radio data of the nucleus are from VLA and VLBI measurements (see text). The near-IR data have been measured from our MAD images.

Compton component is known to be present in this source and to have its maximum power at MeV-GeV energies (Lin et al. 1995; Pian et al. 1996; Hartman et al. 1999; Tavecchio et al. 2007).

The ratio of the inverse Compton to synchrotron luminosities, parameterized by the total radiation density $U_{\text {rad }}^{\prime}$ in the frame of the plasma and by the magnetic energy density $U_{\mathrm{B}}$, respectively, i.e., $L_{I C} / L_{\mathrm{syn}} \simeq U_{\mathrm{rad}} / U_{\mathrm{B}}$ is about 2 for a magnetic field $B=30 \mathrm{G}$ (see Table 3 ), consistent with the multiwavelength spectrum (Pian et al. 1996). In our estimate, which imposes a strong constraint on the magnetic field, we have included the radiation density of the synchrotron photons, of the broad line region (BLR), and of the inner accretion disk, i.e., $U_{\text {int }}^{\prime}, U_{\mathrm{BLR}}^{\prime}$, and $U_{\mathrm{BLR}}^{\prime}$, respectively. The luminosities and radii of both the BLR and disk are taken from Pian et al. (1996). As previously argued by Pian et al. 1996, the largest contribution to the inverse Compton emission is due to SSC, the BLR and accretion-disk seed photons contributing only a small fraction (in total less than 10\%).

\section{The jet and the hotspot}

For the jet knots and hotspot, which are resolved in the radio, optical and near-IR images, we assume an emission region 
Table 4. SED modelling of PKS 0521-365 components.

\begin{tabular}{cccccc}
\hline \hline & $\alpha_{0}^{a}$ & $R_{0}^{b}$ & $n_{0}^{c}$ & $B^{d}$ & $\nu_{\mathrm{c}}^{e}$ \\
\hline nucleus & 0.9 & $1.5 \times 10^{-6}$ & 95000 & 30 & $1.0 \times 10^{13}$ \\
knot A & 0.7 & 0.5 & $1.3 \times 10^{-4}$ & $9.5 \times 10^{-5}$ & $1.0 \times 10^{14}$ \\
knot D & 0.7 & 0.5 & $6.2 \times 10^{-5}$ & $6.9 \times 10^{-5}$ & $5.8 \times 10^{13}$ \\
hotspot & 0.8 & 0.5 & $5.8 \times 10^{-3}$ & $5.3 \times 10^{-4}$ & $3.0 \times 10^{8}$ \\
\hline
\end{tabular}

${ }^{a}$ Spectral index below $v_{\mathrm{c}} ;{ }^{b}$ size of emitting region in $\mathrm{kpc} ;{ }^{c}$ plasma density, i.e. number of particles per $\mathrm{cm}^{3} ;{ }^{d}$ magnetic field in Gauss; ${ }^{e}$ cooling frequency in $\mathrm{Hz}$.

radius of $0.5 \mathrm{kpc}$. Since we do not detect any evidence of an inverse Compton component in the X-ray emission of knot A and hotspot (see also Birkinshaw et al. 2002), we have determined the magnetic field imposing that the inverse Compton luminosity is lower than $10 \%$ of the synchrotron luminosity. The electron density is much lower than that in the nucleus (see Table 4), which is consistent with the presumably more rarefied medium in the external regions of the jet with respect to the core.

For knots D, E and B we can study the SED using only the radio, near-IR, and optical fluxes. The lack of X-ray fluxes thus makes the modelling of their SEDs more uncertain. A curved synchrotron model, with a negligible inverse Compton contribution, accounts well for the data, and in particular for the spectral curvature above the optical frequencies (that cannot be formally reconciled with the $\Delta \alpha=0.5$ expected from simple homogeneous synchrotron radiation) and with the lack of X-ray detection (Birkinshaw et al. 2002). In Table 4, we report only the model parameters for knot $\mathrm{D}$, those of knots $\mathrm{B}$ and $\mathrm{E}$ being very similar.

\section{Comparison between the SED of the core and knot A}

As appears from Fig. 7, the multiwavelength spectrum of the nucleus differs markedly from that of knot A. In particular, the $\mathrm{X}$-ray spectral shapes differ clearly: that of knot A is consistent with being the high energy portion of the synchrotron component that reproduces the radio-to-optical emission, while the $\mathrm{X}$-ray spectrum of the nucleus is caused by an extra emission component that we identify with inverse Compton scattering of the relativistic particles that produce the radio-to-infrared synchrotron spectrum off synchrotron and/or external photons. This difference, which is mainly related to the higher $(\sim 10)$ cooling frequency of knot A with respect to the jet apex. The density of the synchrotron photons in the core is much higher than in knot A (the total luminosity of knot $\mathrm{A}$ is a factor 100 less than in the nucleus and the emission region size is at least 4 orders of magnitude larger). This causes a more efficient electron cooling in the nuclear region via synchrotron self-Compton. The spectrum of PKS0521-365 exhibits prominent broad optical and UV emission lines (e.g. Scarpa et al. 1995) and therefore the density of the photon-field external to the jet within the BLR radius (a fraction of a parsec) is not negligible, while it is irrelevant at the distance of knot A (few kpc). Although electron cooling via inverse Compton off the external photons in the nucleus is not dominant with respect to synchrotron self-Compton, it contributes to making the cooling more efficient (see Ghisellini et al. 1998). On the other hand, knot A is located beyond the BLR (its distance from the nucleus is about 1-2 kpc; well beyond the BLR region), so that its emitting electrons do not cool as efficiently as in the nucleus, and the synchrotron component extends up to the X-rays, with a far less significant contribution from inverse Compton scattering.

\subsection{Radio morphology of the source}

A comparison between the VLBI data (see Sect. 5.1) and the present images confirms that the source is oriented at a relatively large angle with respect to the line of sight and that beaming is not playing a major role. This is suggested by the good agreement between the jet orientation on both the parsec and the kpc scale, and by the extended structure visible at low frequency. The $15 \mathrm{GHz}$ image shows that the arcsecond jet is still clearly onesided. From the jet brightness ratio in the full resolution $15 \mathrm{GHz}$ image, we infer that the jet is still relativistic on the arcsecond scale, with a velocity $>0.79 c$ in the inner kpc region and $>0.3 c$ at $5-6 \mathrm{kpc}$ from the core.

Assuming an average jet velocity $\sim 0.5 \mathrm{c}$ and a constant orientation with respect to the line of sight, we can derive the expected ratio of the approaching to the receding size. The length of the approaching jet should be at least 3 times longer than the receding one. This could explain the lack of a hot spot structure on the jet side. The hot spot should be almost at the end of the radio structure visible at $1.4 \mathrm{GHz}$. At this distance, the ISM might have a lower density and therefore the ISM-jet interaction is not expected to produce this structure. We further note that the kpc scale jet is clearly defined and stops sharply well within the more extended lobe visible at 15 and at $1.4 \mathrm{GHz}$ extent, and this morphology cannot be created by sensitivity problems.

A possible explanation for the difference in morphology between $1.4 \mathrm{GHz}$ and $15 \mathrm{GHz}$ is a reactivation of this source. According to this interpretation, the visible jet is due to the more recent activity, while the more extended structure is related to an older radio activity. In this scenario the eastern hot spot would no longer be connected to the shorter recent counter-jet (not visible because of relativistic effects), and the western hot spot could be missing due to the older source age in this region.

\section{Summary and conclusions}

We have presented high resolution near-IR images of the jet of the nearly BL Lac object PKS 0521-365 using an innovative adaptive-optics device (MAD) built as a demonstrator for multi-conjugated AO imaging. These new data, together with a re-analysis of previous optical (by HST) and radio (by VLA) data, have provided us with insight into a number of remarkable features that are associated with this nearby extragalactic source. The main results from this study are: i) the morphology of the jet is very similar at radio, near-IR, and optical frequencies; ii) the emission from the jet knots is dominated by the synchrotron component, while in the nucleus a significant inverse Compton component is present at high energies; iii) we discovered the near-IR counterpart of the radio hotspot and found its flux to be consistent with the synchrotron emission from radio to X-ray; iv) the resolved red object aligned with the optical jet has no radio counterpart and is thus likely to be a background galaxy that is not associated with the jet.

There are two observations that may clarify the nature of this peculiar system. A spectrum of the red tip appears feasible and may confirm that it is a galaxy. Since the distance of the object is small, proper motion of the order of 0.1 mas of the jet structures are expected, and in principle should be detectable.

In addition to the specific results for the target, these observations exemplify the capabilities of MCAO observations of extragalactic extended sources.

Acknowledgements. E.P. acknowledges support from the Italian Space Agency and the National Institute of Astrophysics INAF through grants ASI-INAF 
I/023/05/0 and ASI I/088/06/0. We wish to thank P. Amico for her support during the MAD observations and the ESO staff at Paranal for kind assistance.

\section{References}

Birkinshaw, M., Worrall, D. M., \& Hardcastle, M. J. 2002, MNRAS, 335, 142 Bono, G., Calamida, A., Corsi, C. E., et al. 2009, in Science with the VLT in the ELT Era, Astrophysics and Space Science Proceedings (Netherlands: Springer), 67

Cardelli, J. A., Clayton, G. C., \& Mathis, J. S. 1989, ApJ, 345, 245

Danziger I. J., Fosbury R. A. E., Goss W. M., \& Ekers R. D. 1979 MNRAS 188, 415

Danziger, I. J., Bergeron, J., Fosbury, R. A. E., et al. 1983, MNRAS, 203, 565

Dreher, J. W., \& Simkin, S. M. 1986, AJ, 91, 58

Falomo, R. 1994, The Messenger, 77, 49

Falomo, R., Scarpa, R., Treves, A., \& Urry, C. M. 2000, ApJ, 542, 731

Ghisellini, G., Celotti, A., Fossati, G., Maraschi, L., \& Comastri, A. 1998, MNRAS, 301, 451

Giroletti, M., Giovannini, G., Taylor, G. B., \& Falomo, R. 2004, ApJ, 613, 752

Jester, S., Meisenheimer, K., Martel, A. R., Perlman, E. S., \& Sparks, W. B. 2007, MNRAS, 380, 828

Hardcastle 2001, A\&A, 373, 881

Hartman, R. C., Bertsch, D. L., \& Bloom, S. D., et al. 1999, ApJS, 123, 79

Harris, D. E., Biretta, J. A., Junor, W., et al. 2003, ApJ, 586, L41

Harris, D. E., Cheung, C. C., Biretta, J. A., et al. 2006, ApJ, 640, 211

Homan, D. C., Ojhia, R., Wardle, J. F. C., et al. 2001, ApJ, 549, 840

Keel, W. C. 1986, ApJ, 302, 296

Lin, Y. C., Bertsch, D. L., Dingus, B. L., et al. 1995, ApJ, 442, 96

Macchetto F., Albrecht, R., Barbieri, C., et al. 1991 ApJ, 369, L55

Marshall, H. L., Harris, D. E., Grimes, J. P., et al. 2001, ApJ, 549, L167

Marshall, H. L., Schwartz, D. A., Lovell, J. E. J., et al. 2005, ApJS, 156, 13
Meisenheimer, K., Yates, M. G., \& Roeser, H.-J. 1997, A\&A, 325, 57 Moretti, A., Piotto, G., Arcidiacono, C., et al. 2009, A\&A, 493, 539 Perlman, E. S., Harris, D. E., Biretta, J. A., Sparks, W. B., \& Macchetto, F. D. 2003, ApJ, 599, L65

Pian, E., Falomo, R., Ghisellini, G., et al. 1996, ApJ, 459, 169

Prieto, M., Brunetti, G., \& Mack, K.-H. 2003, 47, 663

Mack, K.-H., Prieto, M., Almudena, B.; Gianfranco, M., et al. 2003 in New Astron. Rev. 47, 659

Ragazzoni, R. 1996, Journ. of Mod. Opt., 43, 289

Ragazzoni, R. 2000, ESO proceedings of the Backaskog workshop on extremely large telescopes, 57, 175

Ragazzoni, R., Farinato, J., \& Marchetti, E. 2000, SPIE, 4007, 1076

Sambruna, R. M., Maraschi, L., Tavecchio, F., et al. 2002, ApJ, 571, 206

Sambruna, R. M., Gambill, J. K., Maraschi, L., et al. 2004, ApJ, 608, 698

Sambruna, R. M., Donato, D., Tavecchio, F., et al. 2007, ApJ, 670, 74

Sambruna, R. M., Donato, D., Cheung, C. C., Tavecchio, F., \& Maraschi, L. 2008, ApJ, 684, 862

Sari, R., Piran, T., \& Narayan, R. 1998, ApJ, 497, L17

Scarpa, R., Falomo, R., \& Pian E. 1995, A\&A, 303, 730

Scarpa, R., Urry, C. M., Falomo, R., \& Treves, A. 1999, ApJ, 526, 643

Scarpa, R., Urry, C. M., Falomo, R., Pesce, J. E. \& Treves, A. 2000, ApJ, 532, 740

Schlegel, D. J., Imbiber, D. P., \& Davis, M. 1998, ApJ, 500, 525

Schwartz, D. A., Marshall, H. L., Lovell, J. E. J., et al. 2000, ApJ, 540, L69

Sparks, W. B., Biretta, J. A., \& Macchetto, F. 1994, ApJS, 90, 909

Tavecchio, F., Maraschi, L., Wolter, A., et al. 2007, ApJ, 662, 900

Tingay, S. J., \& Edwards, P. G. 2002, AJ, 124, 652

Tingay, S. J., Edwards, P. G., Costa, M. E., et al. 1996, ApJ, 464, 170

Tingay, S. J., Murphy, D. W., \& Edwards, P. G. 1998, ApJ, 500, 673

Ulrich, M. E. 1981, AA 103, L1

Urry, C. M., \& Padovani, P. 1995, PASP, 107803

Urry, C. M., Scarpa, R., O'Dowd, M., et al. 2000, ApJ, 532, 816 